\title{
Hematological abnormalities in patients with malaria and typhoid in Tamale Metropolis of Ghana
}

\author{
Nsoh Godwin Anabire', Paul Armah Aryee ${ }^{2}$ and Gideon Kofi Helegbe ${ }^{1 *}$
}

\begin{abstract}
Objective: Anemia, Leukopenia, and thrombocytopenia are commonly observed hematological abnormalities in malaria and typhoid patients. In this study, we evaluated the prevalence of cytopenias in patients with monoinfections of plasmodium parasites (malaria group) or salmonella bacteria (typhoid group). Full blood counts from 79 patients (age ranging from 18 to 77 years) categorized into malaria and typhoid groups at the Tamale Central Hospital were assessed.

Results: Data generated were entered and analyzed using SPSS version 20 and Graphpad Prism 6. Values were observed to be significant at $p<0.05$. The prevalence of cytopenias were; $29.6,48.0 \%$ for anemia, 38.9, 12.0\% for thrombocytopenia, 20.4, 12.0\% for leukopenia, 13.0, 8.0\% for bicytopenia and 5.6, 4.0\% for pancytopenia in both malaria and typhoid groups respectively. Between the two groups of patients, thrombocytopenia was significantly associated with those in the malaria group $\left(x^{2}=5.84, p<0.016\right)$. No association was found between cytopenias and gender in patients in the malaria group; however, the middle aged group, 36-55 years, was significantly associated with anemia $\left(x^{2}=12.97, p<0.002\right)$. Cytopenias were not associated with gender, and with different age categories in patients in the typhoid group.
\end{abstract}

Keywords: Cytopenias, Malaria, Typhoid, Tamale metropolis

\section{Introduction}

Cytopenias are blood cell abnormalities that result from reduction in the major hematopoietic cell lines such as red blood cells causing anemia, leukocytes causing leukopenia and thrombocytes causing thrombocytopenia. Bicytopenia occurs where there is a reduction below reference ranges in any two of the major cell lines [1]. The simultaneous presence of anemia, thrombocytopenia and leukopenia in a person is termed pancytopenia.

Anemia [2-5], leukopenia [6-9] and thrombocytopenia [10-16] are commonly presented in plasmodium and salmonella infections. The occurrence of Cytopenias may be attributed to bone marrow suppression and

\footnotetext{
*Correspondence: ghelegbe@uds.edu.gh

1 Department of Biochemistry \& Molecular Medicine, School of Medicine and Health Sciences, University for Development Studies, P. O. Box TL 1883, Tamale, Ghana

Full list of author information is available at the end of the article
}

hemo-phagocytosis $[13,17]$. Elsewhere, 94/61.3\% anemia, 70/40\% thrombocytopenia and 12/4\% leukopenia have been reported among adults with malaria or typhoid respectively $[14,18,19]$.

Bicytopenia and pancytopenia usually result from direct or indirect decreasing effect on hematopoietic cell production in the bone marrow [20-22]. These kinds of cytopenias are not uncommon in malaria; bone marrow diagnosis of adults with bicytopenia and pancytopenia has shown that $3 \%$ of bicytopenia and $6 \%$ of pancytopenia were caused by malaria [23, 24]. Even though bone marrow studies have shown no clear explanation for the peripheral blood pancytopenia in typhoid fever [25], a case report of severe pancytopenia in an adult was attributed to hemo-phagocytosis [26].

Despite many global studies reporting anemia, thrombocytopenia and leukopenia with malaria and typhoid in adults, limited studies of these cytopenias exist in Ghana. The studies in Ghana, are mostly on anemia in children 
[27-30] and in pregnant women [31-34]. Thus, this study was intended to determine the prevalence and association of cytopenias in adults with plasmodium or salmonella infections in Tamale.

\section{Main text \\ Methods \\ Study area}

The study was conducted in the Tamale metropolis; the Northern Regional capital of Ghana. The metropolis has $13 \%$ of the total land area of the Northern Region (70, 383 square $\mathrm{km}$ ), and a total population of 223,252 comprising 111,109 males and 112,143 females [35].

\section{Study design, study site and population}

A hospital based cross-sectional study was conducted from February to April 2015 on patients with monoinfections of plasmodium parasites or salmonella bacteria between the ages of 18-77 years at Tamale central Hospital. Tamale Central Hospital is one of the northern regional hospitals and serves nearly 240,000 people with numerous referrals from other districts in northern Ghana.

\section{Sample size and exclusion criteria}

Sample size was obtained based on the number of adults that showed up with either malaria or typhoid within the period of sampling. Pregnant women and individuals diagnosed with helminthiasis, sickle cell disease, and kidney disease were excluded from this study.

\section{Data collection and processing}

Demographic data (including sex and age) of each study participant was collected using a standardized questionnaire (Additional file 1), after consent was obtained from participants. Enumerators were given a one-day training prior to data collection. The questionnaire was pretested by random administration to 20 patients. Age was categorized as follows: young adults (18-35), middle aged adults (36-55) and the aged (above 55) [36]. Clinical diagnoses including sickle cell disease, kidney disease, helminthiasis and urine pregnancy tests results were collected from the patients' folders by the clinicians on duty.

A Vacutainer $\mathrm{K}_{3}$ EDTA tube (Anhui Kangning Industrial group, Tianchang, China, Catologue Number: VG4000515) was used to collect $3 \mathrm{ml}$ of venous blood from each participant for laboratory investigation. The blood samples were collected by a trained medical laboratory scientist.

Malaria was diagnosed using CareStart ${ }^{\mathrm{TM}}$ HRP-2 rapid diagnostic test (Access Bio Inc., New Jersey, USA, Catalogue number: G0141). The testing, results reading and interpretations were performed by strictly following the manufacturer's protocol. The presence of malaria parasites was confirmed by microscopy using thick blood smears which were stained with $20 \%$ Giemsa solution at $\mathrm{pH} 7.2$ [37]. The reading of the blood smears and interpretation of the results were done as previously reported [38].

The slide agglutination method previously described [39] was performed for typhoid using commercially available Widal kits (Lab Care Diagnostics, Mumbai, India, Catalogue number: CW0111). Briefly, patients' plasma was titrated in the following volumes $40,20,10$ and $5 \mu \mathrm{l}$. One plate was designated for antigen-O and the other antigen-H. Later, one drop of antigen-O and antigen- $\mathrm{H}$ of Salmonella typhi were added to patient's plasma distributed on the respective plates. This was then mixed and rocked and agglutination observed and interpreted as previously reported [39].

Hb-Hemoglobin, WBC-White blood cell and PLTPlatelets were analyzed using the automated blood cell analyzer Sysmex XS-500i (Sysmex Corporation, Kobe, Japan). Anaemia was diagnosed by $\mathrm{Hb}<12 \mathrm{~g} / \mathrm{dl}$ for nonpregnant females and $\mathrm{Hb}<13 \mathrm{~g} / \mathrm{dl}$ for males [40], while PLT count $<150 \times 10^{9} / 1$ and WBC count $<4.0 \times 10^{9} / 1$ were used respectively to determine thrombocytopenia and leukopenia [41]. Bicytopenia was determined if a subject had a combination of any 2 cytopenias and pancytopenia as simultaneously having all three forms of cytopenia.

\section{Statistical analysis}

Data were analyzed using SPSS Version 20 (IBM Corporation, Chicago, USA) and Graphpad Prism 6 (GraphPad Software Inc., San Diego, USA). All test statistics with $\mathrm{p}$ values $<0.05$ were considered as statistically significant. Continuous variables were described using median and interquartile range (IQR), and compared by Mann-Whitney test, whilst categorical variables were presented as counts and percentages, and compared by Fisher's exact test or Pearson's $\chi^{2}$ test.

\section{Results \\ General characteristics of the study participants}

Out of the 79 individuals recruited for this study, majority, 55 (69.6\%) were females (Table 1). The median age was 31 years (IQR 21-47 years) with majority of the study participants, 47 (59.5\%) being young adults (1835 years), Table 1 . The median hemoglobin count was $12.8 \mathrm{~g} / \mathrm{dl}$ (IQR $11.3-14.1 \mathrm{~g} / \mathrm{dl}$ ), whilst the median platelet and white cell counts were $213 \times 10^{3} / \mu \mathrm{L}$ (IQR 142.0 $287 \times 10^{3} / \mu \mathrm{L}$ ) and $5.8 \times 10^{3} / \mu \mathrm{L}$ (IQR $4.5-7.0 \times 10^{3} / \mu \mathrm{L}$ ) respectively. 
Table 1 Socio-demographic characteristics and hematological profile among malaria and typhoid patients

\begin{tabular}{|c|c|c|c|}
\hline Characteristics & Malaria group $(N=54)$ & Typhoid group $(N=25)$ & $p$ value* $(95 \% \mathrm{Cl})$ \\
\hline Age, years, median (IQR) & $28(20-45)$ & $37(24-54)$ & $0.1544(-2.000$ to 14.000$)$ \\
\hline \multicolumn{4}{|l|}{ Age category, years, $n(\%)$} \\
\hline $18-35$ & $36(66.7)$ & $11(44.0)$ & \multirow[t]{3}{*}{0.069 (0.371 to 1.530$)$} \\
\hline $36-55$ & $9(16.7)$ & $10(40.0)$ & \\
\hline $56+$ & $9(16.7)$ & $4(16.0)$ & \\
\hline \multicolumn{4}{|l|}{ Gender, $n(\%)$} \\
\hline Male & $14(25.9)$ & $10(40.0)$ & \multirow[t]{2}{*}{0.206 (0.192 to 1.435$)$} \\
\hline Female & $40(74.1)$ & $15(60.0)$ & \\
\hline Hemoglobin, median (IQR) & $13.3(11.8-14.3)$ & $12.6(11.1-13.3)$ & $0.0986(-1.800$ to 0.200$)$ \\
\hline Platelets, median (IQR) & $197.5(120.5-273.5)$ & $284.0(175.5-320.0)$ & 0.0065 (20.00 to 118.00) \\
\hline White blood cells, median (IQR) & $5.7(4.3-6.6)$ & $6.2(4.9-9.8)$ & $0.2301(-0.410$ to 1.760$)$ \\
\hline Anemia, $n(\%)$ & $16(29.6)$ & $12(48.0)$ & $0.112(-0.431$ to 0.411$)$ \\
\hline Thrombocytopenia, $n$ (\%) & $21(38.9)$ & $3(12.0)$ & $0.016(1.241$ to 17.550$)$ \\
\hline Leukopenia, $n$ (\%) & $11(20.4)$ & $3(12.0)$ & 0.530 (0.474 to 7.430$)$ \\
\hline Bicytopenia, $n(\%)$ & $7(13.0)$ & $2(8.0)$ & $0.711(0.392$ to 8.911$)$ \\
\hline Pancytopenia, $n$ (\%) & $3(5.6)$ & $1(4.0)$ & 1.000 (0.139 to 14.300$)$ \\
\hline
\end{tabular}

Source: Field Survey. *Analyzed using Mann-Whitney test and Pearson's $x^{2}$ test or Fisher's exact test. Two tailed $p$ value significant at $p<0.05$

Malaria group patients with plasmodium mono-infection, Typhoid group patients with salmonella mono-infection

\section{Prevalence and association of cytopenias in patients with malaria or typhoid}

The prevalence of cytopenias were; $29.6,48.0 \%$ for anemia, 38.9, $12.0 \%$ for thrombocytopenia, 20.4, $12.0 \%$ for leukopenia, 13.0, 8.0\% for bicytopenia and 5.6, 4.0\% for pancytopenia in patients with mono-infections of plasmodium and salmonella respectively (Table 1). While thrombocytopenia was significantly associated $(p<0.016)$, with patients in the malaria group, anaemia, leukopenia, bicytopenia and pancytopenia were independent of either groups (Table 1). Similar levels of $\mathrm{Hb}$ and leucocytes were observed between both groups, however, levels of platelets were statistically lower, $p=0.007$ in the malaria group compared to the typhoid group (Table 1). Pancytopenia was only found in females (7.5\% in malaria and $6.7 \%$ in typhoid), Table 2. Cytopenias were observed to be independent of gender and most age categories for either groups, however, the middle aged malaria group, was significantly associated with anemia $(p=0.002)$, Table 2 .

\section{Discussion}

Malaria and typhoid are quite common in Tamale because the prevailing warm and humid climate is conducive for the infection, concomitant with poor sanitary practices that promote the infections [42]. Malaria endemicity is nearly the same across the year but peaks slightly in the rainy season [43]. Despite the frequent diagnoses of anemia, thrombocytopenia and leukopenia in patients presenting with malaria or typhoid at the health facilities, not much is known about the prevalence of these cytopenias among adults in Ghana; most especially in northern Ghana. Thus, providing the impetus for this study in Tamale. To the best of our knowledge, we are, for the first time, reporting the prevalence of cytopenias in patients with malaria or typhoid in Ghana.

In the current study, thrombocytopenia was found to be the most prevalent in the adults with plasmodium infection. This contradicts 2 studies $[18,19]$ that showed anemia as the most prevalent cytopenia in malaria patients. However, both studies were conducted in south Asia where the dominant malaria parasite $(P$. vivax) is different from that in Ghana (P. falciparum). Unlike P. vivax, $P$. falciparum is very virulent; it multiples rapidly in the blood and has the ability to sequester into diverse host organs [44]. It has been shown that some P. falciparum infected-erythrocytes can bind platelets to form plateletmediated clumps [45] and postulations are that immune mediated lysis of these clumps cause thrombocytopenia in $P$. falciparum infection. Thus, differences in parasite strains could be a possible reason for the difference seen in this study; more so because [19] realized a significant increase in the incidence of thrombocytopenia in $P$. falciparum cases than in $P$. vivax cases. Our findings of a higher prevalence and significant association of thrombocytopenia with malaria adds to studies indicating that thrombocytopenia is an indicator of malaria in the adult population [46]. 
Table 2 Association between cytopenias; and gender and age groups in malaria $(\mathbf{N}=54)$ and typhoid patients $(\mathrm{N}=25)$

\begin{tabular}{|c|c|c|c|c|c|}
\hline Characteristics & ${ }^{a}$ Malaria group & ${ }^{\beta}$ Typhoid group & ${ }^{a}$ Malaria group & ${ }^{\beta}$ Typhoid group & $p$ value* $(95 \% \mathrm{Cl})$ \\
\hline & Anemia & & & & \\
\hline & Yes, $n(\%)$ & & No, $n(\%)$ & & \\
\hline Male & $4(28.6)$ & $6(60.0)$ & $10(71.4)$ & $4(40.0)$ & ${ }^{a} 1.000(0.244-3.574),{ }^{\beta} 0.428(0.439-11.530)$ \\
\hline Female & $12(30.0)$ & $6(40.0)$ & $28(70.0)$ & $9(60.0)$ & \\
\hline $18-35$ & $6(16.7)$ & $6(54.5)$ & $30(83.3)$ & $5(45.5)$ & ${ }^{a} 0.002(0.009-0.346),{ }^{\beta} 0.798(0.318-10.210)$ \\
\hline $36-55$ & $7(77.8)$ & $4(40.0)$ & $2(22.2)$ & $6(60.0)$ & \\
\hline \multirow[t]{3}{*}{$56+$} & $3(33.3)$ & $2(50.0)$ & $6(66.7)$ & $2(50.0)$ & \\
\hline & Thrombocytopenia & & & & \\
\hline & Yes, $n(\%)$ & & No, $n(\%)$ & & \\
\hline Male & $6(42.9)$ & $0(0.0)$ & $8(57.1)$ & $10(100.0)$ & ${ }^{a} 0.723(0.363-4.308),{ }^{\beta} 0.051(0.003-1.409)$ \\
\hline Female & $15(37.5)$ & $6(40.0)$ & $25(62.5)$ & $9(60.0)$ & \\
\hline $18-35$ & $14(38.9)$ & $3(27.3)$ & $22(61.1)$ & $8(72.7)$ & ${ }^{a} 0.349(0.027-12.300),{ }^{\beta} 0.114(0.390-3.772)$ \\
\hline $36-55$ & $2(22.2)$ & $0(0.0)$ & $7(77.8)$ & $10(100.0)$ & \\
\hline \multirow[t]{3}{*}{$56+$} & $5(55.6)$ & $0(0.0)$ & $4(44.4)$ & $4(100.0)$ & \\
\hline & Leukopenia & & & & \\
\hline & Yes, $n(\%)$ & & No, $n(\%)$ & & \\
\hline Male & $2(14.3)$ & $1(10.0)$ & $12(85.7)$ & $9(90.0)$ & ${ }^{a} 0.708(0.108-3.053),{ }^{\beta} 0.179(0.017-1.680)$ \\
\hline Female & $9(22.5)$ & $6(40.0)$ & $31(77.5)$ & $9(60.0)$ & \\
\hline $18-35$ & $8(22.2)$ & $2(18.2)$ & $28(77.8)$ & $9(81.8)$ & ${ }^{a} 0.752(0.248-21.110),{ }^{\beta} 0.612(0.153-3.360)$ \\
\hline $36-55$ & $2(22.2)$ & $1(10.0)$ & $7(77.8)$ & $9(90.0)$ & \\
\hline \multirow[t]{3}{*}{$56+$} & $1(11.1)$ & $0(0.0)$ & $8(88.9)$ & $4(100.0)$ & \\
\hline & Bicytopenia & & & & \\
\hline & Yes, $n(\%)$ & & No, $n(\%)$ & & \\
\hline Male & $3(21.4)$ & $1(10.0)$ & $11(78.6)$ & $9(90.0)$ & ${ }^{a} 0.358(0.475-12.690),{ }^{\beta} 1.000(0.106-12.460)$ \\
\hline Female & $4(10.0)$ & $1(6.7)$ & $36(90.0)$ & $14(93.3)$ & \\
\hline $18-35$ & $2(5.6)$ & $1(9.1)$ & $34(94.4)$ & $10(90.9)$ & ${ }^{a} 0.057(0.016-0.860),{ }^{\beta} 0.811(0.048-2.076)$ \\
\hline $36-55$ & $2(22.2)$ & $1(10.0)$ & $7(77.8)$ & $9(90.0)$ & \\
\hline \multirow[t]{3}{*}{$56+$} & $3(33.3)$ & $0(0.0)$ & $6(66.7)$ & $4(100.0)$ & \\
\hline & Pancytopenia & & & & \\
\hline & Yes, $n(\%)$ & & No, $n(\%)$ & & \\
\hline Male & $0(0.0)$ & $0(0.0)$ & $14(100.0)$ & $10(100.0)$ & ${ }^{a} 0.560(0.018-7.611),{ }^{\beta} 1.000(0.017-12.460)$ \\
\hline Female & $3(7.5)$ & $1(6.7)$ & $37(92.5)$ & $14(93.3)$ & \\
\hline $18-35$ & $2(5.6)$ & $1(9.1)$ & $34(94.4)$ & $10(90.9)$ & ${ }^{a} 0.589(0.038-5.858),{ }^{\beta} 0.811(0.048-2.076)$ \\
\hline $36-55$ & $1(11.1)$ & $1(10.0)$ & $8(88.9)$ & $9(90.0)$ & \\
\hline $56+$ & $0(0.0)$ & $0(0.0)$ & $9(100.0)$ & $4(100.0)$ & \\
\hline
\end{tabular}

Source: Field Survey. *Analyzed using Pearson's $x^{2}$ test or Fisher's exact test, ${ }^{a}$ and ${ }^{\beta}$ represent $p$ values for association of cytopenias with gender and with age groups in Malaria group: patients with plasmodium mono-infection, and Typhoid group: patients with salmonella mono-infection, respectively. Two tailed $p$ value significant at $p<0.05 .95 \% \mathrm{Cl} 95 \%$ confidence interval

While extensive studies have been conducted on the occurrence of anemia in children and pregnant women with malaria [2-5], little is known of such studies in non-pregnant female and male adults. In areas with high malaria transmission, adults usually develop semi-immunity to malaria due to exposure to several different clones of malaria parasites. As such, adults are mostly asymptomatic, with low parasite loads that could lead to anemia and its related complications. In this study, anemia was strongly associated with the middle aged group, 35-55 years but was, however, gender independent. Even though it has been shown that anemia in malaria patients differ in different ages [17], it is not clear why anemia is significantly associated with this particular age group. Nevertheless, it is tempting to speculate that immune reaction is very active in this age group resulting in high loss of uninfected RBCs as well as the infected ones [47]. This should prompt a closer look at anemia with malaria infection in this age category in Tamale. 
Even though leukopenia was the least prevalent cytopenia, its prevalence was quite significant, and could confound the estimation of malaria parasite density in adults in Tamale based on the WHO criteria of WBC count of 8000 cells $/ \mu \mathrm{L}$.

The high prevalence of anemia recorded in the adults with typhoid is similar to a study by [14]. This finding could be attributed to myeloid maturation arrest and decrease in the number of erythroblasts as shown by [13]. The independence of the cytopenias with typhoid, age and gender among the study population may suggest that $\mathrm{Hb}$, PLT and WBCs counts may not be good hematological markers for the diagnosis of typhoid in adults in Tamale.

Pancytopenia and bicytopenia are a common hematological problem encountered in clinical practice, which have multiple causes and the underlying pathology determines the management and prognosis of the patients. Bicytopenia and pancytopenia were observed in both infections; and the knowledge that both cytopenias are seen in quite a number of malaria and typhoid patients in Tamale is important information that would help in timely diagnosis and proper management of such cases, leading to earlier discharge of the patient. Unlike many studies that had male dominance in pancytopenia cases [48-51], we realized that pancytopenia was only found in females for either infection, which could possibly be attributed to the female dominance in this study. This falls in line with what pertains in Ghana, where women are more likely to access health care. More to the point, women often have to ask for permission from their husbands in order to access treatment for themselves, which may delay depending on whether their husbands or family elders would agree [52]. This may explain why in this study, the worse form of cytopenia, pancytopenia is only found in females, because the longer it takes for one to get an appropriate treatment for these infections, the more damage the disease causes to one's hematopoietic system.

\section{Conclusion}

The high prevalence and association of thrombocytopenia with malaria in adults in Tamale buttresses numerous findings of low platelets count as a reliable diagnostic marker for malaria in adult populations in Africa. The association of anemia with the middle aged adults with malaria should prompt a closer look at anemia with plasmodium infection in this age category in Tamale. Our finding of pancytopenia in only females, should prompt strengthening of education on barriers preventing females from accessing healthcare delivery.

\section{Limitation}

This study was conducted within a relatively shorter period and the sample size was small. Since malaria endemicity is nearly constant within the metropolis, an all year investigation of these cytopenias in patients with malaria or typhoid would be important in substantiating the findings in this study.

\section{Additional files}

Additional file 1. Document used in the collection of socio-demographic and health data. This is a blank document which contains the questionnaire used in the collection of socio-demographic characteristics, and the health records of the study participants.

Additional file 2. Data set of the subjects. This is a SPSS document containing data transribed from the questionnaire.

\section{Abbreviations}

Hb: hemoglobin; WBCs: white blood cells; PLT: platelets; IQR: interquartile range; RBCs: red blood cells; WHO: World Health Organization.

\section{Authors' contributions}

GKH and PAA conceived and designed the study. Questionnaire administration, laboratory analysis and data entering were performed by NGA. NGA, GKH and PAA analyzed the data. NGA drafted the manuscript with GKH and PAA.

GKH supervised the study. All authors read and approved the final manuscript.

\section{Author details}

${ }^{1}$ Department of Biochemistry \& Molecular Medicine, School of Medicine and Health Sciences, University for Development Studies, P. O. Box TL 1883, Tamale, Ghana. ${ }^{2}$ Department of Nutritional Sciences, School of Allied Health Sciences, University for Development Studies, P. O. Box TL 1883, Tamale, Ghana.

\section{Acknowledgements}

We extend our heartfelt gratitude to the numerous clinicians at Tamale central Hospital for their enormous support. Again we thank the staff at registry and patients' folders unit, and the staff at the hematology unit of the Tamale Central Hospital for their immeasurable efforts. We also thank all our study participants for their patience and agreeing to be part of this study.

\section{Competing interests}

The authors declare that they have no competing interests.

\section{Availability of data and materials}

The datasets supporting the findings of this article are available in this manuscript (Additional file 2).

\section{Consent for publication \\ Not applicable.}

\section{Ethics approval and consent to participate}

Ethical approval for the study was obtained from the School of Medicine and Health Sciences (SMHS) and the School of Allied Health Sciences (SAHS) joint ethical review board of the University for Development Studies, Ghana. Written informed consent was provided by each study participants.

\section{Funding}

Not applicable.

\section{Publisher's Note}

Springer Nature remains neutral with regard to jurisdictional claims in published maps and institutional affiliations. 
Received: 3 November 2017 Accepted: 31 May 2018

Published online: 05 June 2018

\section{References}

1. Beck N. Pancytopenia and bicytopenia. In: Beck N, editor. Diagnostic hematology. New York: Springer; 2009. p. 297-308.

2. Awandare GA, et al. Increased levels of inflammatory mediators in children with severe Plasmodium falciparum malaria with respiratory distress. J Infect Dis. 2006;194(10):1438-46.

3. Helegbe GK, et al. Complement activation in Ghanaian children with severe Plasmodium falciparum malaria. Malar J. 2007;6(1):165.

4. Ugwu EO, et al. Malaria and anaemia in pregnancy: a cross-sectional study of pregnant women in rural communities of Southeastern Nigeria. Int Health. 2014;6(2):130-7.

5. Douamba Z, et al. Asymptomatic malaria correlates with anaemia in pregnant women at Ouagadougou, Burkina Faso. BioMed Res Int. 2012

6. McKenzie FE, et al. White blood cell counts and malaria. J Infect Dis. 2005;192(2):323-30.

7. Alves-Junior ER, et al. Assumed white blood cell count of $8000 \mathrm{cells} / \mu \mathrm{L}$ overestimates malaria parasite density in the Brazilian Amazon. PLoS ONE. 2014;9(4):e94193.

8. Mallouh AA, Sa'di AR. White blood cells and bone marrow in typhoid fever. Pediatr Infect Dis J. 1987;6(6):527-8.

9. Abdool GM, et al. The white cell count in typhoid fever. Trop Geogr Med 1992;44(1-2):23-7.

10. Lacerda MVG, et al. Thrombocytopenia in malaria: who cares? Mem Inst Oswaldo Cruz. 2011;106:52-63.

11. Horstmann R, et al. Malaria-induced thrombocytopenia. Ann Hematol. 1981:42(3):157-64.

12. Barber $B E$, et al. A prospective comparative study of knowlesi, falciparum, and vivax malaria in Sabah, Malaysia: high proportion with severe disease from Plasmodium knowlesi and Plasmodium vivax but no mortality with early referral and artesunate therapy. Clin Infect Dis. 2012;56(3):383-97.

13. Khosla $\mathrm{S}$, et al. Haematological profile in typhoid fever. Trop Doct. 1995;25(4):156-8.

14. Abro AH, et al. Hematological and biochemical changes in typhoid fever. Pak J Med Sci. 2009;25(2):166-71.

15. Shrivastava K, Vahikar S, Mishra V. Hematological profile in typhoid fever. Trop J Pathol Microbiol. 2015;1(01)

16. Dangana A, Ajobiewe J, Nuhu A, Haematological changes associated with Salmonella typhi and Salmonella paratyphi in humans. Int J Biomed Health Sci. 2010;6(4)

17. Menendez C, Fleming A, Alonso P. Malaria-related anaemia. Parasitol Today. 2000;16(11):469-76.

18. Akhtar $\mathrm{S}$, et al. Hematological changes in malaria: a comparative study. IOSR-JPBS. 2012;2:15-9.

19. Farogh Azfar, et al. Haematological abnormalities in malaria. Biomedica. 2009;25(52-5):5.

20. Bates I, Bain BJ. 23 Approach to the diagnosis and classification of blood diseases. 2006.

21. Kar M, Ghosh A. Pancytopenia. J Indian Acad Clin Med. 2002;3:29-34.

22. Mudenge B, et al. Pancytopenia in Zimbabwe. Am J Med Sci. 1999;317(1):22-32.

23. Durrani SH, et al. Incidentally diagnosed bicytopenia showing a wide spectrum of pathologies on bone marrow morphology. KJMS 2015;8(2):247.

24. Barik S, Chandoke RK, Verma AK. A prospective clinico-hematological study in 100 cases of pancytopenia in capital city of India. J Appl Hematol. 2014;5(2):45.

25. Getta HA. The role of the bone marrow study in typhoid fever with pancytopenia. Zanco J Med Sci. 2010;14:3.

26. Sood R, Roy S, Kaushik P. Typhoid fever with severe pancytopenia. Postgrad Med J. 1997;73(855):41.

27. Iqbal SA, et al. Hematological differences among malaria patients in rural and Urban Ghana. J Trop Pediatr. 2016;62(6):477-86.
28. Koram KA, et al. Seasonal profiles of malaria infection, anaemia, and bednet use among age groups and communities in northern Ghana. Tropical Med Int Health. 2003;8(9):793-802.

29. Mockenhaupt FP, et al. Manifestation and outcome of severe malaria in children in northern Ghana. Am J Trop Med Hyg. 2004;71(2):167-72.

30. Squire DS, et al. Effect of Plasmodium falciparum malaria parasites on haematological parameters in Ghanaian children. J Parasit Dis. 2016;40(2):303-11.

31. Mockenhaupt FP, et al. Anaemia in pregnant Ghanaian women: importance of malaria, iron deficiency, and haemoglobinopathies. Trans R Soc Trop Med Hyg. 2000;94(5):477-83.

32. Tay SC, et al. Malaria and anaemia in pregnant and non-pregnant women of child-bearing age at the University Hospital. Ghana: Kumasi; 2013.

33. Tay SCK, Nani EA, Walana W. Parasitic infections and maternal anaemia among expectant mothers in the Dangme East District of Ghana. BMC Res Notes. 2017:10(1):3.

34. Clerk CA, et al. The epidemiology of malaria among pregnant women attending antenatal clinics in an area with intense and highly seasonal malaria transmission in northern Ghana. Trop Med Int Health. 2009:14(6):688-95.

35. Ghanadistricts.com. Northern: Tamale metropolis. http://ghanadistricts. gov.gh/About-District-Details.aspx?distID=139\&distName=Tamale\%20 Metropolitan. Accessed 25 Apr 2017

36. Petry NM. A comparison of young, middle-aged, and older adult treatment-seeking pathological gamblers. Gerontologist. 2002;42(1):92-9.

37. Njunda A, et al. Comparison of capillary and venous blood using blood film microscopy in the detection of malaria parasites: a hospital based study. Sci J Microbiol. 2013;2(5):89-94.

38. Kwenti $T E$, et al. Identification of the plasmodium species in clinical samples from children residing in five epidemiological strata of malaria in Cameroon. Trop Med Health. 2017;45(1):14.

39. Andualem $\mathrm{G}$, et al. A comparative study of Widal test with blood culture in the diagnosis of typhoid fever in febrile patients. BMC Res Notes. 2014;7(1):653

40. World Health Organization. Haemoglobin concentrations for the diagnosis of anaemia and assessment of severity. 2011. http://www.who.int/ vmnis/indicators/haemoglobin.pdf. 2015

41. Hoffbrand AV, et al. Postgraduate hematology. 6th ed. Hoboken: Blackwell Publishing Ltd.; 2011

42. Napari PN, Cobbinah PB. Environmental Sanitation Dilemma in the Tamale Metropolis, Ghana. J Sust Dev. 2004;6.

43. Owusu-Agyei S, et al. Epidemiology of malaria in the forest-savanna transitional zone of Ghana. Malar J. 2009;8(1):220

44. Miller $\mathrm{LH}$, et al. The pathogenic basis of malaria. Nature. 2002:415(6872):673-9.

45. Pain A, et al. Platelet-mediated clumping of Plasmodium falciparuminfected erythrocytes is a common adhesive phenotype and is associated with severe malaria. Proc Natl Acad Sci. 2001;98(4):1805-10.

46. Khan SJ, Abbass Y, Marwat MA. Thrombocytopenia as an indicator of malaria in adult population. Malar Res Treat. 2012;2012.

47. Helegbe GK, et al. Rate of red blood cell destruction varies in different strains of mice infected with Plasmodium berghei-ANKA after chronic exposure. Malar J. 2009;8(1):91.

48. Kumar R, et al. Pancytopenia-a six year study. J Assoc Phys India. 2001:49:1078-81.

49. Tilak V, Jain R. Pancytopenia-a clinico-hematologic analysis of 77 cases. Indian J Pathol Microbiol. 1999;42(4):399-404.

50. Khunger JM, et al. Pancytopenia-a clinico haematological study of 200 cases. Indian J Pathol Microbiol. 2002;45(3):375-9.

51. Khodke $\mathrm{K}$, et al. Bone marrow examination in cases of pancytopenia. $J$ Indian Acad Clin Med. 2001;2:1-2.

52. Tolhurst R, Nyonator FK. Looking within the household: gender roles and responses to malaria in Ghana 2006;100(4):321-6. 